\title{
A CATAlogue OF THE BEES OF CALIFORNiA.
}

\author{
BY T. D. A. COCKERELL, EAST LAS VEGAS, N. MEX.
}

In the Report of the Agricultural experiment station of the University of California for 1898-1901, recently published, Mr. Carroll Fowler gives an account of the long tongued bees of California. This is a very useful paper, so far as it goes, and Mr. Fowler has been able to make important additions to our knowledge of California bees. Unfortunately, however, the paper was prepared in 1899, and has not been brought up to date; consequently it omits numerous species recorded since that year, and is not altogether modern in the matter of generic nomenclature. It also omits several species published before 1899 , and in a good many cases the names of species are incorrectly spelled. In some cases, I am inclined to doubt the identification of the species, but unless I have actual knowledge to the contrary, I must for the present treat all published records as correct.

The list here presented includes also the short-tongued bees of California, for completeness' sake. It need hardly be said, that we know comparatively little about California bees; it is quite certain that any student who takes up this group in California will find very numerous new species. The list now offered is intended to be of service to such a student or students, as it is hoped that the publication of Mr. Fowler's paper will have created an interest in the bees.

\section{Colletes Latr.}

americana Cr., La Jolla. californica Prov.

Prosopis Fabr. (cf. Entom., i898, p. 218. ) polifolii Ckll., Mt. Lowe. coquillettii Ckll., Los Angeles Co. bakeri Ckll.

ruidosensis Ckll., var.

suffusa Ckll., var.

tridentula Ckll. mesillae Ck1l., Los Angeles Co. varifrons $\mathbf{C r}$.

Halictus Latr.

californicus (Prov.).

farinosus $\mathrm{Sm}$.

titusi Crawf., San Diego. Allied to H. trizonatus. gracilis Rob.

tegularis Rob. (belongs to Chloralictu Rob.). armaticeps $\mathrm{Cr}$.

SPHecodes Latr.

mandibularis Cr., San Pedro.

Agapostemon Sm.

femoratus Crawf.

californicus Crawf., San Pedro, etc. texanus Cr., San Pedro.

\section{Andrena Fabr.}

knuthiana Ck1l., Berkeley.

nigra Prov.

nigripes Prov.

phenax Ckll., only two submarginal cells. foxii Ckll., only two submarginal cells. chalybaea (Cr.), only two submarginal cells. 
Diandrena is a new subgenus or genus for the blue species of California with only two submarginal cells; type chalybaea, described as a Panurgus. Here also belongs $A$. foxii, and, I think, A. phenax.

\section{Parandrena Rob.}

regularis (Cr.).

concinnula Ck1l., So. Cal.

enocki Ck1l., So. Cal.

\section{Hesperapis Ckll.}

eumorpha (Ck11.), near Los Angeles (Davidson).

\section{Halictoides Nyl.}

saundersi Ckll.

mulleri Ckll.

virgatus Ckll.

davidsoni Ckll., San Gabriel Mts.

Micrandrena Ashm.

pacifica Ashm. (a smallish black species with

a large reddish stigma; detailed description not published yet).

\section{Nomia Latr.}

Two undescribed species from California are in the National museum, bearing MS. names by Ashmead. One is near bakeri; the other near foxii but larger, though not nearly so large as nortoni.

Spinoliella Ashm. (cf. Ann. mag. nat. hist. Jan. 1901, p. 129).

edwardsii (Cr.).

scutellaris (Fowler).

visaliensis (Fowler).

anthidius (Fowler).

zonalis (Cr.).

cincta (Cr.).

Calliopsis Sm.

californicus $\mathrm{Cr}$.

obscurellus Cr.
Panurginus Nyl.

cressoniellus Ckll.

albitarsis (Cr.).

Hylaeosoma Ashm. (cf. Ann. mag. nat. hist. July, I902, p. 42).

atriceps $(\mathrm{Cr}$.$) .$

\section{Perdita Sim.}

claypolei Ckll., Mt. Lowe.

rhois Ckll., San Diego.

rhois reducta Ckll., San Diego.

californica (Cr.).

interrupta $\mathrm{Cr}$.

trisignata $\mathrm{Ck} l l$.

Nomada Fabr.

citrina $\mathrm{Cr}$.

interruptella Fowler.

obliquella Fowler.

obscurella Fowler.

rivalis $\mathrm{Cr}$.

suavis $\mathrm{Cr}$.

rubrica Prov. (Fowler makes this a var. of

bisignata, but Robertson has shown that

bisignata cannot be certainly recognized).

crotchii Cr.

edwardsii $\mathrm{Cr}$.

melliventris $\mathrm{Cr}$.

opposita $\mathrm{Cr}$.

vinnula $\mathrm{Cr}$.

civilis $\mathrm{Cr}$.

flavipes Prov.

erythraea Dalla Torre (rubra Prov.).

fragilis $\mathrm{Cr}$.

vincta Say.

lepida $\mathrm{Cr}$.

bisignata "Say," Fowler.

formula Viereck, San Pedro.

Neopasites Ashm.

fulviventris (Cr.).

EPeOLus Latr.

compactus Cr.

nigriceps Sm. 
occidentalis $\mathrm{Cr}$. superbus Prov. californicus $\mathrm{Cr}$. faceatus Prov.

Triepeolus Rob.

concavus (Cr.).

Melecta Latr.

californica $\mathrm{Cr}$.

Zacosmia Ashm.

maculata (Cr.), San Pedro.

Bombomelecta Patt.

edwardsii (Cr.).

separata $(\mathrm{Cr}$.$) .$

thoracica (Cr.).

\section{Stelis Panz.}

laticincta $\mathrm{Cr}$.

sexmaculata Ashm.

\section{Osmia Panz.}

californica $\mathrm{Cr}$.

cobaltina $\mathrm{Cr}$.

maura $\mathrm{Cr}$.

quadriceps $\mathrm{Cr}$.

exigua $\mathrm{Cr}$.

atriventris $\mathrm{Cr}$.

dubia $\mathrm{Cr}$.

purpurea $\mathrm{Cr}$.

lignaria Say.

nigrifrons $\mathrm{Cr}$.

brevis $\mathrm{Cr}$.

albiventris $\mathrm{Cr}$.

rustica "Cr." Fowler (Robertson says that rustica is identical with albiventris).

Monumetha Cr. (genus hardly distinct from Osmia).

imperfecta Prov.

\section{Heriades Spin.}

odontura Ck1l., near Los Angeles. glaucum Fowler. albicinctum Prov., probably an Ashmeadiella. semirubra Ck1l., not a true Heriades.

\section{Ashmeadiella Ckll.}

californica (Ashm.), described under Chalicodoma.

Alcidamea Cr.

uvulalis Ckll., Mojave Desert. simplex (Cr.) (producta Cr.).

Andronicus Cr.

hesperius Ckll., sp. nov. $\delta$. About $12 \mathrm{~mm}$. long, black, closely punctured; wings smoky; flagellun brown beneath; tarsi with short reddish hair. Clypeus with the anterior border thickened and faintly concave; scape stout; flagellum like that of AlcidaMEA, but not hooked at the tip; first ventral segment of abdomen produced into a backwardly directed spine at the middle of its posterior margin; seventh dorsal segment subtruncate with rounded edges. Rock Creek, Mojave Desert.

\section{Chelostoma Latr.}

californicum $\mathrm{Cr}$.

australe Ckll., near Los Angeles.

\section{Anthidium Fabr.}

atriventre $\mathrm{Cr}$.

emarginatum Say.

maculosum $\mathrm{Cr}$.

tricuspidum Prov.

collectum Huard (compactum Prov.).

californicum $\mathrm{Cr}$.

edwardsii Cr.

palliventre $\mathrm{Cr}$.

illustre $\mathrm{Cr}$.

maculifrons (Sm.?), Fowler.

Dianthidium Ckll.

consimile (Ashm.).

ehrhorni (Ckll.), Mojave Desert.

Megachile Latr.

occidentalis leucotricha Ckll., near Los Angeles. 
fidelis $\mathrm{Cr}$.

fidelis concinnula Ckll.

davidsoni Ckll., near Los Angeles.

angelarum Ckll.

vidua $\mathrm{Sm}$. (frigida $\mathrm{Sm}$.).

latimanus Say.

pugnata Say.

montivaga $\mathrm{Cr}$.

brevis Say.

exilis Cr. (studiosa Cr.).

frugalis Cr., San Gabriel Mts., Rock Creek, Mojave Desert (Davidson), near Los Angeles (Davidson).

manifesta Cr., var. Banning, Riverside Co.

pruina Sm., Catalina Island and near Los

Angeles (Davidson).

\section{Lithurgopsis Fox.}

apicalis (Cr.), var. opuntiae Ckll., $\delta$, Palm Springs, Colorado Desert (Davidson).

Ceratina Latr.

dupla Say.

tejonensis $\mathrm{Cr}$.

acantha Prov.

arizonensis Ckll., Mt. Lowe.

Melissodes Latr.

actuosa $\mathrm{Cr}$.

californica $\mathrm{Cr}$.

personatella Ckll., La Jolla.

lupina $\mathrm{Cr}$.

nigrifrons $\mathrm{Cr}$.

stretchii $\mathrm{Cr}$.

olivacea Cr. ("Appears to be related to the genus Ancyloscelis in neuration and length of tongue; however, the $\delta$ has the clypeus yellow; the abdomen in both sexes is covered with a dense appressed pubescence like the male of Eutechnia grisella, and egg-shaped; the claws are blunt and cleft in both sexes."-H. L. Viereck, litt. I901).

menuacha $\mathrm{Cr}$.

tepida $\mathrm{Cr}$.

obliqua Say.

montana Cr., San Pedro, La Jolla.
Diarasia Patt.

bituberculata (Cr.), described under Melisso-

des. Mr. Viereck tells me it is a Diadasia. nerea Fowler.

cinerea Fowler.

enavata $\mathrm{Cr}$.

albovestita Prov.

tricincta Prov. (Fowler says this is enavata). rinconis opuntiae Ckll., San Pedro.

friesei Ck1l., So. Cal.

australis Cr., San Bernardino.

\section{Xenoglossa Sm.}

strenua (Cr.) (cucurbitarum Ckll.).

patricia angustior Ckll., Los Angeles.

\section{Peponapis Rob.}

angelica Ckll. (pruinosa Fowler, not Say).

Synhalonia Patt.

edwardsii (Cr.).

californica Fowler.

albicans Prov.

albopilosa Fowler.

frater (Cr.) (speciosa $\mathrm{Cr}$. ).

acerba (Cr.).

nevadensis (Cr.) (intrudens $\mathrm{Cr}$.).

EMpHORopsis Ashm.

depressus (Fowler).

miserabilis (Cr.).

floridanus (Sm.).

There is also an undescribed species which Ashmead made the type of Meliturgopsis.

Anthophora Latr.

edwardsii Cr.

pacifica $\mathrm{Cr}$.

urbana $\mathrm{Cr}$.

californica $\mathrm{Cr}$.

crotchii $\mathrm{Cr}$.

catalinae Ckll., Catalina I.

exigua $\mathrm{Cr}$.

maculifrons $\mathrm{Cr}$.

quinquefasciata Prov.

flavocincta Huard (nigrocincta Prov.). 

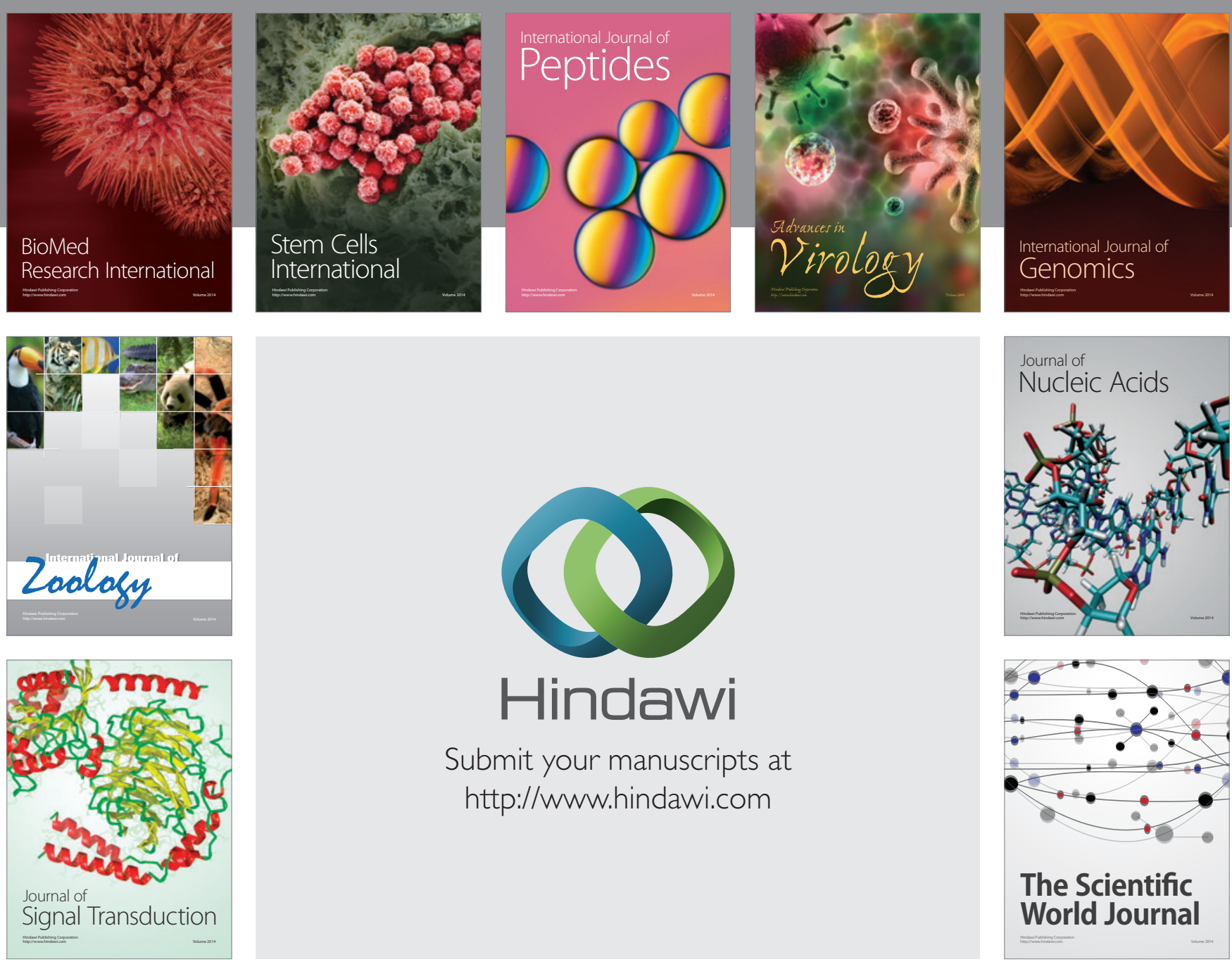

Submit your manuscripts at

http://www.hindawi.com
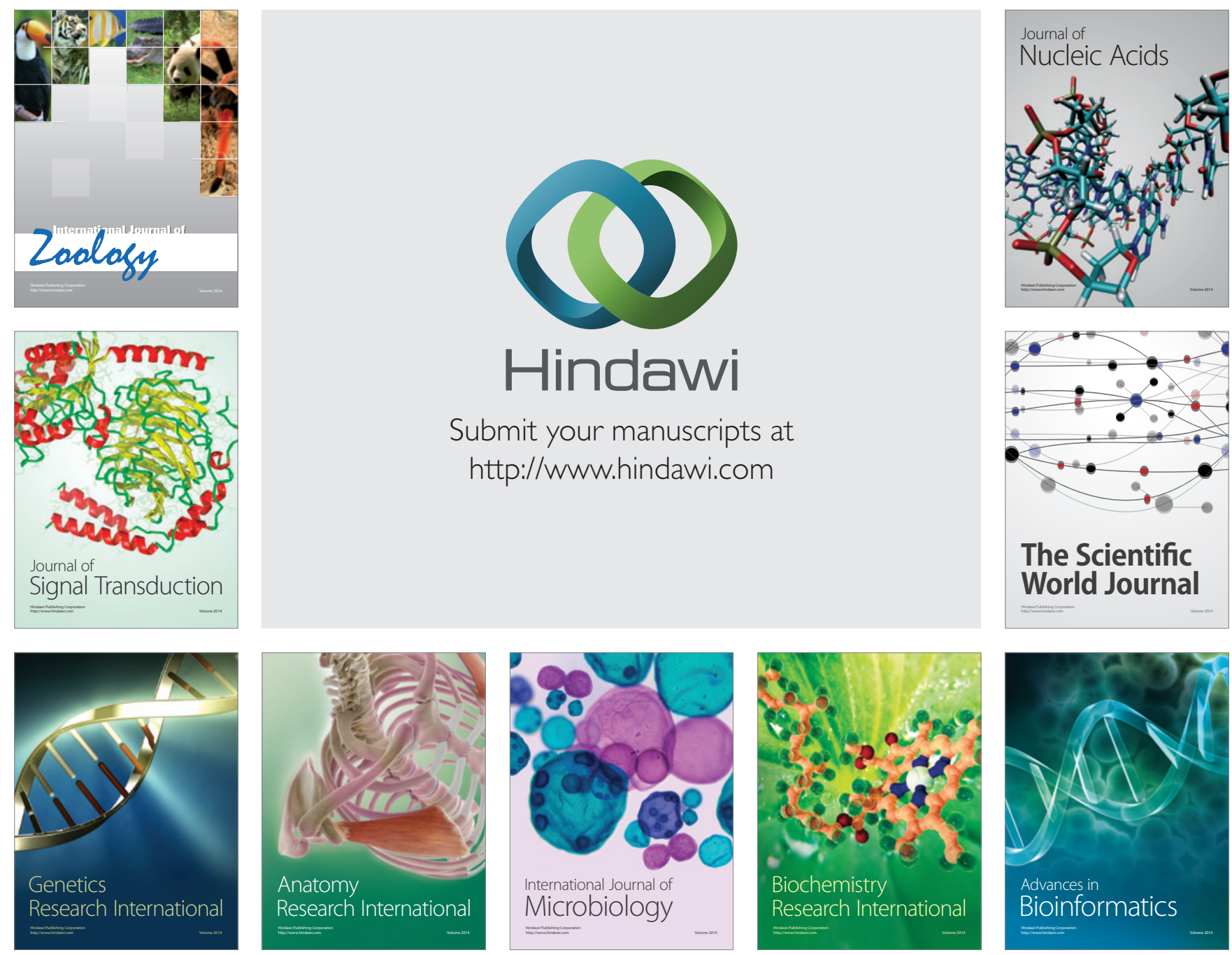

The Scientific World Journal
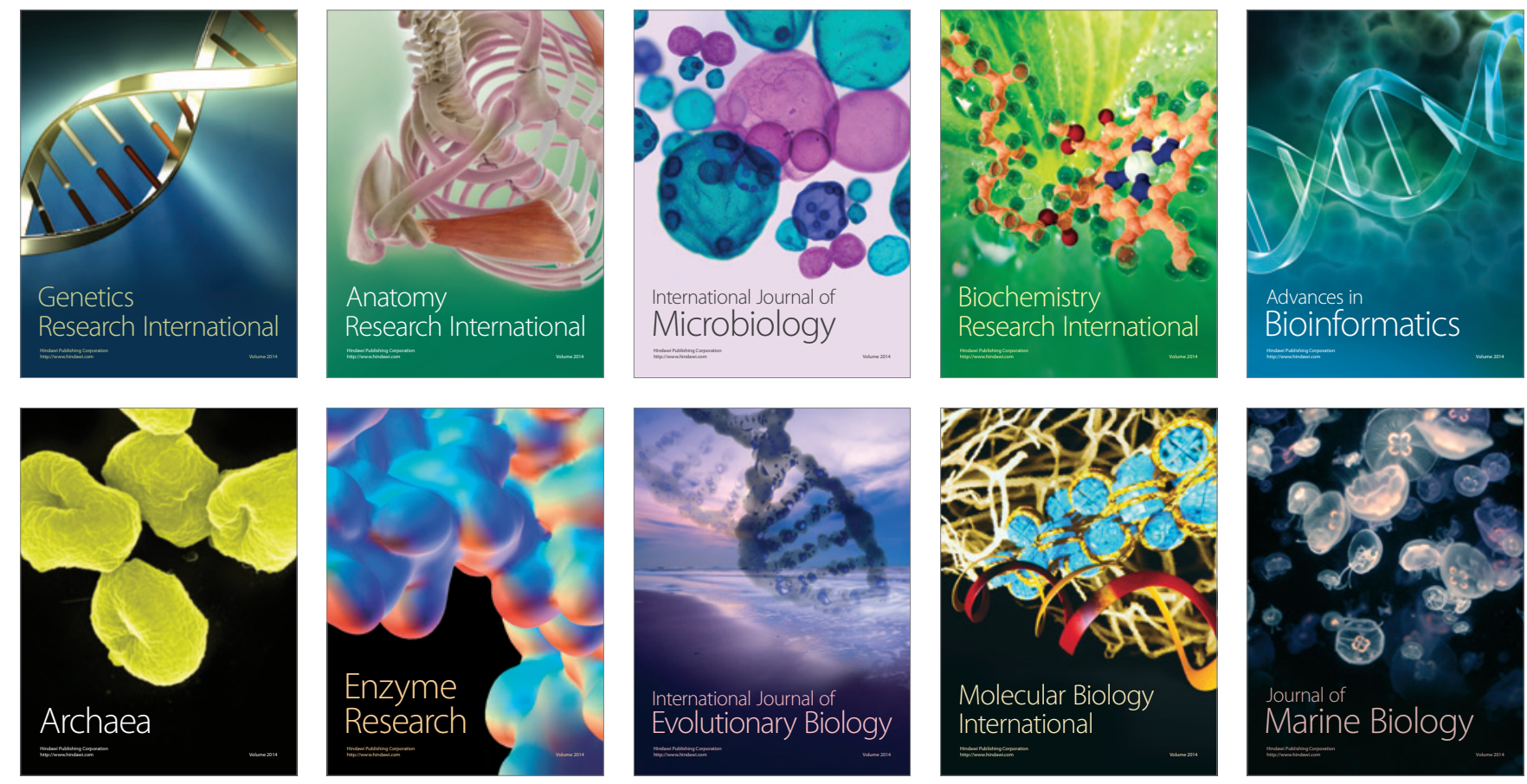